\title{
Erratum to: The whole genome sequence of the Mediterranean fruit fly, Ceratitis capitata (Wiedemann), reveals insights into the biology and adaptive evolution of a highly invasive pest species
}

Alexie Papanicolaou', Marc F. Schetelig², Peter Arensburger ${ }^{3}$, Peter W. Atkinson 4,5, Joshua B. Benoit ${ }^{6}$, Kostas Bourtzis ${ }^{7,8}$, Pedro Castañera ${ }^{9}$, John P. Cavanaugh ${ }^{6}$, Hsu Chao $^{10}$, Christopher Childers $^{11}$, Ingrid Curril ${ }^{12}$, Huyen Dinh ${ }^{10}$, HarshaVardhan Doddapaneni ${ }^{10}$, Amanda Dolan ${ }^{13}$, Shannon Dugan ${ }^{10}$, Markus Friedrich ${ }^{14}$, Giuliano Gasperi ${ }^{15}$, Scott Geib ${ }^{16}$, Georgios Georgakilas ${ }^{17}$, Richard A. Gibbs ${ }^{10}$, Sarah D. Giers ${ }^{18}$, Ludvik M. Gomulski ${ }^{15}$, Miguel González-Guzmán ${ }^{9}$, Ana Guillem-Amat ${ }^{9}$, Yi $\operatorname{Han}^{10}{ }^{10}$ Artemis G. Hatzigeorgiou ${ }^{17}$, Pedro Hernández-Crespo ${ }^{9}$, Daniel S. T. Hughes ${ }^{10}$, Jeffery W. Jones ${ }^{19}$, Dimitra Karagkouni ${ }^{17}$, Panagiota Koskinioti ${ }^{20}$, Sandra L. Lee ${ }^{10}$, Anna R. Malacrida ${ }^{15}$, Mosè Manni ${ }^{15}$, Kostas Mathiopoulos ${ }^{20}$, Angela Meccariello ${ }^{21}$, Monica Munoz-Torres ${ }^{22}$, Shwetha C. Murali ${ }^{10}$, Terence D. Murphy ${ }^{23}$, Donna M. Muzny ${ }^{10}$, Georg Oberhofer ${ }^{12}$, Félix Ortego ${ }^{9}$, Maria D. Paraskevopoulou ${ }^{17}$, Monica Poelchau ${ }^{11}$, Jiaxin Qu ${ }^{10}$, Martin Reczko ${ }^{24}$, Hugh M. Robertson ${ }^{18}$, Andrew J. Rosendale ${ }^{6}$, Andrew E. Rosselot ${ }^{6}$, Giuseppe Saccone ${ }^{21}$, Marco Salvemini ${ }^{21}$, Grazia Savini ${ }^{15}$, Patrick Schreiner ${ }^{5}$, Francesca Scolari ${ }^{15}$, Paolo Siciliano ${ }^{15}$, Sheina B. Sim ${ }^{16}$, George Tsiamis ${ }^{8}$, Enric Ureña ${ }^{9}$, loannis S.Vlachos ${ }^{17}$, John H. Werren ${ }^{13}$, Ernst A. Wimmer ${ }^{12}$, Kim C. Worley ${ }^{10}$, Antigone Zacharopoulou ${ }^{25}$, Stephen Richards ${ }^{10}$ and Alfred M. Handler ${ }^{26^{*}}$

\section{Erratum}

After publication of our recent article [1] we noticed that Monica Munoz-Torres had been omitted from the author list. We have now added her, and the updated Funding and Authors' contributions sections are below.

\section{Funding}

Support of this project was provided by the U.S. Department of Agriculture (USDA), Agricultural Research Service (ARS), Animal and Plant Health Inspection Service (APHIS), and National Institute of Food and Agriculture (NIFA)-Biotechnology Risk Assessment Grants Program (grant \#2011-39211-30769 to $\mathrm{AMH}$ ) for funding the initial phase of this project, and to the National Institutes of Health (NIH)-National Human Genome Research Institute (NHGRI) for funding the medfly genome sequencing, assembly and Maker 2.0 automated annotation as part of the i5K 30 genome pilot project (grant \#U54 HG003273 to RAG). The NIH Intramural Research Program, National Library of Medicine funded the NCBI Gnomon annotation and the USDA-National Agricultural Library (NAL) provided support for the WebApollo curation website, with support for manual curation training (to MM-T) provided by NIGMS (grant \#5R01GM080203),

\footnotetext{
* Correspondence: al.handler@ars.usda.gov

${ }^{26}$ USDA-ARS, Center for Medical, Agricultural and Veterinary Entomology, 1700 S.W. 23rd Drive, Gainesville, FL 32608, USA

Full list of author information is available at the end of the article
}

NHGRI (grant \#5R01HG004483), and the U.S. Department of Energy (contract \#DE-AC02-05CH11231). Support was provided for: toxin metabolism and insecticide resistance gene studies from MINECO, Spain (AGL2013-42632-R to FO and PH-C); microRNAs, horizontal gene transfer and bacterial contaminant studies from the European Social Fund and National Strategic Reference Framework-THALES (MIS375869 to $K B, G T, A G H$, and $K M$ ) and the U.S. National Science Foundation (DEB 1257053 to JHW); cuticle protein gene studies from USDA-NIFA (grant \#2016-67012-24652 to AJR); sex-determination studies from L.R. Campania (grant 5/02, 2008 to GS); male reproduction and sexual differentiation studies from the FAO/IAEA (Technical Contract No: 16966 to GGa) and Cariplo IMPROVE (to FS); and programmed cell death gene studies and genomic data analysis (to MFS) from the Emmy Noether program, DFG (SCHE 1833/1-1) and the LOEWE Center for Insect Biotechnology \& Bioresources grant of the Hessen State Ministry of Higher Education, Research and the Arts (HMWK), Germany and from the USDA-NIFA-Biotechnology Risk Assessment Grants Program (grant \#2015-33522-24094 to AMH).
Authors' contributions
$\mathrm{AMH}$ and SR conceived of and directed the project whose management was assisted by MFS, AP, and RAG. LMG reared the inbred ISPRA lines and extracted genomic DNA and RNA that was used for library construction, sequencing, assembly and Maker annotations at HGSC by SR, SD, SLL, HC, HVD, HD, YH, JQ, SCM, DSTH, KCW, DMM and RAG. Additional community RNA-Seq analysis was performed by GO, IC, and EAW; JAMg annotations were performed by AP and Gnomon (NCBI) annotations were directed by TDM that were provided for manual annotation at the USDA-NAL WebApollo 
site managed by MP, and CC, with curation assistance and training by MM-T; $A D, J H W, A P, T D M, G T$ and $K B$ identified and analyzed bacterial sequence contaminants; SG and SBS performed orthology and phylogeny analysis; $\mathrm{AMH}, \mathrm{AZ}$, and $\mathrm{PH}-\mathrm{C}$ made scaffold map assignments; GT and KB performed symbiont horizontal gene transfer analysis; PS, PA, PWA and AMH analyzed transposable elements; GGe, DK, MDP, ISV, and AGH manually annotated and analyzed microRNAs; LMG, FS, GS, PS, MM, ARM, and GGa manually annotated and analyzed odorant binding protein, odorant receptor, and seminal fluid protein genes; SDG and HMR manually annotated and analyzed gustatory and ionotrophic receptor genes; MF and JWJ manually annotated and analyzed opsin genes; AJR, AER, JPC and JBB manually annotated and analyzed aquaporin and cuticle protein genes; PK, MR and KM manually annotated and analyzed immunity-related genes; PC, FO, PH-C, MG-G, EU and AG-A manually annotated and analyzed toxin metabolism and insecticide resistance genes (P450s, carboxylesterases, GSTs, cysLGICs and sodium channels); MS and GS manually annotated and analyzed sex determination genes; and MFS and AMH manually annotated and analyzed programmed cell death genes. All authors read and approved the final manuscript.

\section{Author details}

${ }^{1}$ Hawkesbury Institute for the Environment, Western Sydney University,

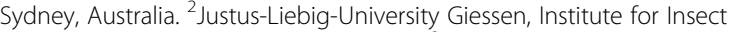
Biotechnology, 35394 Giessen, Germany. ${ }^{3}$ Department of Biological Sciences, Cal Poly Pomona, Pomona, CA 91768, USA. ${ }^{4}$ Department of Entomology and Center for Disease Vector Research, University of California Riverside, Riverside, CA 92521, USA. ${ }^{5}$ Interdepartmental Graduate Program in Genetics, Genomics \& Bioinformatics, University of California Riverside, Riverside, CA 92521, USA. ${ }^{6}$ Department of Biological Sciences, University of Cincinnati, Cincinnati, OH 45221, USA. ${ }^{7}$ Insect Pest Control Laboratory, Joint FAO/IAEA Programme of Nuclear Techniques in Food and Agriculture, Seibersdorf, Vienna, Austria. ${ }^{8}$ Department of Environmental and Natural Resources Management, University of Patras, Agrinio, Greece. ${ }^{9}$ Department of Environmental Biology, Centro de Investigaciones Biológicas, CSIC, 28040 Madrid, Spain. ${ }^{10}$ Human Genome Sequencing Center, Department of Human and Molecular Genetics, Baylor College of Medicine, 77030 Houston, TX, USA. ${ }^{11}$ National Agricultural Library, USDA, 20705 Beltsville, MD, USA.

${ }^{12}$ Georg-August-Universität Göttingen, Johann-Friedrich-Blumenbach-Institut für Zoologie und Anthropologie, 37077 Göttingen, Germany. ${ }^{13}$ Department of Biology, University of Rochester, 14627 Rochester, NY, USA. ${ }^{14}$ Department of Biological Sciences, Wayne State University, 48202 Detroit, MI, USA.

${ }^{15}$ Department of Biology and Biotechnology, University of Pavia, 27100 Pavia, Italy. ${ }^{16}$ USDA-ARS, Pacific Basin Agricultural Research Center, 96720 Hilo, HI, USA. ${ }^{17}$ DIANA-Lab, Department of Electrical \& Computer Engineering, University of Thessaly, 38221 Volos, Greece and Hellenic Pasteur Institute, 11521 Athens, Greece. ${ }^{18}$ Department of Entomology, University of Illinois at Urbana-Champaign, 61801 Urbana, IL, USA. ${ }^{19}$ Department of Biological Sciences, Oakland University, 48309 Rochester, MI, USA. ${ }^{20}$ Department of Biochemistry and Biotechnology, University of Thessaly, Larissa, Greece.

${ }^{21}$ Department of Biology, University of Naples Federico II, 80126 Naples, Italy. ${ }^{22}$ Environmental Genomics and Systems Biology Division, Lawrence Berkeley National Laboratory, 94720 Berkeley, CA, USA. ${ }^{23}$ National Center for Biotechnology Information, National Library of Medicine, National Institutes of Health, 20892 Bethesda, MD, USA. ${ }^{24}$ Institute of Molecular Biology and Genetics, Biomedical Sciences Research Centre "Alexander Fleming", Athens, Greece. ${ }^{25}$ Department of Biology, University of Patras, Patras, Greece.

${ }^{26}$ USDA-ARS, Center for Medical, Agricultural and Veterinary Entomology,

1700 S.W. 23rd Drive, Gainesville, FL 32608, USA.

Received: 12 January 2017 Accepted: 12 January 2017

Published online: 18 January 2017

\section{References}

1. Papanicolaou A, et al. The whole genome sequence of the Mediterranean fruit fly, Ceratitis capitata (Wiedemann), reveals insights into the biology and adaptive evolution of a highly invasive pest species. Genome Biol. 2016;17:192. 